Tohoku Math. J.

66 (2014), 137-153

\title{
EXISTENCE AND NONEXISTENCE OF POSITIVE SOLUTIONS FOR PARAMETRIC NEUMANN PROBLEMS WITH $p$-LAPLACIAN
}

\author{
Dumitru Motreanu, Viorica V. Motreanu* \\ AND NiKOlaOS S. PAPAGEORGIOU
}

(Received July 2, 2012)

\begin{abstract}
Using variational methods based on the critical point theory and suitable truncation and comparison techniques, we study existence, multiplicity and nonexistence of positive solutions for a parametric nonlinear Neumann problem driven by the $p$-Laplacian. Our hypotheses cover the case of nonlinearities of concave-convex type whose exponents depend on the parameter.
\end{abstract}

Introduction. Let $\Omega \subset \boldsymbol{R}^{N}$ be a bounded domain with a $C^{2}$-boundary $\partial \Omega$ and let $p \in(1,+\infty)$. We consider the following parametric nonlinear Neumann problem

$\left(P_{\lambda}\right)$

$$
\begin{cases}-\Delta_{p} u(x)+\beta(x)|u(x)|^{p-2} u(x)=f(x, u(x), \lambda) & \text { in } \Omega, \\ \frac{\partial u}{\partial n_{p}}=0 & \text { on } \partial \Omega,\end{cases}
$$

with the real parameter $\lambda>0$. Here $\Delta_{p}$ denotes the $p$-Laplace differential operator defined by $\Delta_{p} u=\operatorname{div}\left(|\nabla u|^{p-2} \nabla u\right)$ for all $u \in W^{1, p}(\Omega)$ (where $|\cdot|$ stands for the Euclidean $\boldsymbol{R}^{N}$-norm) and the nonlinearity $f(x, s, \lambda)$ is a Carathéodory function (i.e., for all $(s, \lambda) \in \boldsymbol{R} \times(0,+\infty)$, $x \mapsto f(x, s, \lambda)$ is measurable and for a.a. $x \in \Omega,(s, \lambda) \mapsto f(x, s, \lambda)$ is continuous $)$. In the problem, $\partial u / \partial n_{p}:=\gamma_{n}\left(|\nabla u|^{p-2} \nabla u\right) \in W^{-1 / p^{\prime}, p^{\prime}}(\partial \Omega)$ denotes the generalized outward normal derivative (see [8]).

Our aim in this paper is to obtain criteria for the existence, multiplicity and nonexistence of positive solutions for problem $\left(P_{\lambda}\right)$ as the parameter $\lambda>0$ varies. Specifically, the main result of the paper, stated as Theorem 1.3, is a bifurcation-type result for the parametric problem $\left(P_{\lambda}\right)$ ensuring that there exists a value $\hat{\lambda}$ of the parameter such that $\left(P_{\lambda}\right)$ has at least two positive solutions if $\lambda<\hat{\lambda},\left(P_{\hat{\lambda}}\right)$ has at least one positive solution, and $\left(P_{\lambda}\right)$ has no positive solution if $\lambda>\hat{\lambda}$.

The hypotheses under which our result is formulated cover nonlinearities $f(x, \cdot, \lambda)$ in $\left(P_{\lambda}\right)$ which are $(p-1)$-superlinear near $+\infty$ and $(p-1)$-sublinear near $0^{+}$. Some related works focused on the case of concave-convex nonlinearities (see [2], [4], [6], [7], [13]). In this paper, our hypotheses cover this case and also allow to go beyond it. In particular, they

2000 Mathematics Subject Classification. Primary 35J25; Secondary 35J92.

Key words and phrases. p-Laplacian, concave-convex nonlinearities, positive solutions, bifurcation-type theorem.

*Work funded by a Marie Curie Intra-European Fellowship for Career Development within the European Community's 7th Framework Program (Grant Agreement No. PIEF-GA-2010-274519). 
allow nonlinearities of concave-convex type whose exponents depend on the parameter $\lambda$. In Section 4, we propose several examples of nonlinearities which fulfill our hypotheses.

Our approach is variational, based on the critical point theory combined with suitable truncation and comparison techniques. Our proofs are elementary in the sense that they do not need to use maximum principles or Morse theory.

The rest of the paper is organized as follows. In Section 1, we state our hypotheses and formulate our main result. In Section 2, we implement lower and upper solution techniques in the treatment of the parametric nonlinear Neumann problem $\left(P_{\lambda}\right)$. In Section 3, we apply those techniques to the proof of our theorem. Finally, examples of nonlinearities to which our result applies are provided in Section 4.

Acknowledgement. The second author is grateful to Lucas Fresse for useful discussions and for his remarks which allowed to generalize some hypotheses and simplify certain proofs in a previous version of the manuscript.

1. Statement of the main result. In the following, we will use the Banach space $W^{1, p}(\Omega)$ endowed with the usual Sobolev norm $\|\cdot\|$, whereas $|\cdot|$ stands for the Euclidean $\boldsymbol{R}^{N}$-norm. For a given $u \in W^{1, p}(\Omega)$, we will use the notation $u^{ \pm}=\max \{ \pm u, 0\}$. Also, by $|\cdot|_{N}$ we denote the Lebesgue measure on $\boldsymbol{R}^{N}$ and, for $q \in[1,+\infty]$, by $\|\cdot\|_{q}$ we denote the $L^{q}(\Omega)$-norm.

We consider the following hypotheses on the nonlinearity $f(x, s, \lambda)$. Set $F(x, s, \lambda)=$ $\int_{0}^{s} f(x, t, \lambda) d t$.

$\left(\mathrm{H}_{f}\right) f: \Omega \times \boldsymbol{R} \times(0,+\infty) \rightarrow \boldsymbol{R}$ is a Carathéodory function such that $f(x, 0, \lambda)=0$ for a.a. $x \in \Omega$, all $\lambda>0$, and

(i) there exist a function $a:(0,+\infty) \rightarrow(0,+\infty)$ with $\lim _{\lambda \rightarrow 0} a(\lambda)=0$, a function $r:(0,+\infty) \rightarrow\left(p, p^{*}\right)$ with $\inf _{\lambda \in(0,+\infty)} r(\lambda)>p$, and a constant $c>0$ such that

$$
|f(x, s, \lambda)| \leq a(\lambda)+c s^{r(\lambda)-1} \text { for a.a. } x \in \Omega, \text { all } s \geq 0, \lambda>0 ;
$$

(ii) for all $\lambda>0$, we have

$$
\lim _{s \rightarrow+\infty} \frac{f(x, s, \lambda)}{s^{p-1}}=+\infty \quad \text { uniformly for a.a. } x \in \Omega
$$

(iii) for all $\lambda>0$, there exists $\beta_{\lambda}^{*} \in L^{1}(\Omega)_{+}$such that $\lambda \mapsto\left\|\beta_{\lambda}^{*}\right\|_{1}$ is bounded on the compact subsets of $(0,+\infty)$ and

$$
\xi_{\lambda}(x, s) \leq \xi_{\lambda}(x, t)+\beta_{\lambda}^{*}(x) \text { for a.a. } x \in \Omega \text {, all } 0 \leq s \leq t,
$$

where $\xi_{\lambda}(x, s)=f(x, s, \lambda) s-p F(x, s, \lambda)$;

(iv) for all $t>0$ and $\lambda>0$, there exists $\theta(t, \lambda)>0$ with $\theta(t, \lambda) \rightarrow+\infty$ as $\lambda \rightarrow+\infty$ such that

$$
\inf \{f(x, s, \lambda) ; s \geq t\} \geq \theta(t, \lambda) \quad \text { for a.a. } x \in \Omega ;
$$


(v) for all $\lambda>0$, there exist $q=q(\lambda) \in[1, p)$ and $\eta_{0}=\eta_{0}(\lambda)>0$ satisfying

$$
\eta_{0} s^{q-1} \leq f(x, s, \lambda) \text { for a.a. } x \in \Omega \text {, all } s \geq 0 ;
$$

(vi) for all $\lambda>0$ and $\rho>0$, there exists $\sigma_{\rho}=\sigma_{\rho}(\lambda)>0$ such that for a.a. $x \in \Omega$, $s \mapsto f(x, s, \lambda)+\sigma_{\rho} s^{p-1}$ is nondecreasing on $[0, \rho]$;

(vii) for all $t>0$ and $\lambda>\lambda^{\prime}>0$, we can find $\theta_{0}\left(t, \lambda, \lambda^{\prime}\right)>0$ such that

$$
f(x, s, \lambda)-f\left(x, s, \lambda^{\prime}\right) \geq \theta_{0}\left(t, \lambda, \lambda^{\prime}\right) \quad \text { for a.a. } x \in \Omega, \text { all } s \geq t .
$$

REMARK 1.1. (a) Note that hypothesis $\left(\mathrm{H}_{f}\right)$ (iii) is satisfied if for every $\lambda>0$, there exists $M_{\lambda}>0$ such that, for a.a. $x \in \Omega, s \mapsto \xi_{\lambda}(x, s)$ is nondecreasing on $\left[M_{\lambda},+\infty\right)$, and with the assumption that the maps $\lambda \mapsto M_{\lambda}$ and $\lambda \mapsto a(\lambda)$ (in $\left(\mathrm{H}_{f}\right)$ (i)) are bounded on compact sets. Furthermore, a sufficient condition for the latter property is that for a.a. $x \in \Omega$ and all $\lambda>0, s \mapsto f(x, s, \lambda) / s^{p-1}$ is nondecreasing on $\left[M_{\lambda},+\infty\right)$ (reason as in $[10$, Lemma 2.4] taking into account that $f$ has nonnegative values).

(b) We observe that we do not require that $s \mapsto f(x, s, \lambda)$ necessarily satisfies the classical (unilateral) Ambrosetti-Rabinowitz condition (see [3] for $p=2$ and for instance [10] for $p$ general). This condition implies that the nonlinearity has a strong growth at infinity and it guarantees that the $C^{1}$-functional associated to the problem satisfies the Palais-Smale condition. In our setting, instead of the Ambrosetti-Rabinowitz condition, we use the assumption that $f$ is $(p-1)$-superlinear at infinity together with a quasimonotonicity condition on $s \mapsto f(x, s, \lambda) s-p F(x, s, \lambda)$ (see hypotheses $\left(\mathrm{H}_{f}\right)$ (ii) and (iii)). It allows us to incorporate nonlinearities with slower growth near $+\infty$, which do not satisfy the Ambrosetti-Rabinowitz condition (see Example (f) in Section 4). Moreover, we do not need that our functionals satisfy the Palais-Smale condition and we can replace it by the weaker Cerami condition, which is sufficient in our situation.

REMARK 1.2. Since we are looking for positive solutions and hypotheses $\left(\mathrm{H}_{f}\right)$ concern only the positive semiaxis $\boldsymbol{R}_{+}=[0,+\infty)$, without any loss of generality, we will assume that $f(x, s, \lambda)=0$ for a.a. $x \in \Omega$, all $s \leq 0$, all $\lambda>0$.

Typical examples of nonlinearities satisfying $\left(\mathrm{H}_{f}\right)$ are provided in Section 4.

The hypotheses on $\beta$ are the following:

$\left(\mathrm{H}_{\beta}\right) \beta \in L^{\infty}(\Omega), \beta(x) \geq 0$ for a.a. $x \in \Omega, \beta \neq 0$.

By a positive solution for problem $\left(P_{\lambda}\right)$ we mean a function $u \in C^{1}(\bar{\Omega}), u>0$ in $\bar{\Omega}$, which is a (weak) solution of $\left(P_{\lambda}\right)$. The nonlinear Green's identity then ensures that $u$ satisfies the boundary value condition $\partial u / \partial n_{p}=0$ on $\partial \Omega$ (see also Motreanu-Papageorgiou [12, pp. 24-25]). Our main result is the following bifurcation-type theorem for problem $\left(P_{\lambda}\right)$.

THEOREM 1.3. If hypotheses $\left(\mathrm{H}_{f}\right)$ and $\left(\mathrm{H}_{\beta}\right)$ hold, then there exists $\hat{\lambda}>0$ such that (a) for all $\lambda \in(0, \hat{\lambda})$, problem $\left(P_{\lambda}\right)$ has at least two (positive) solutions $u_{0}, \tilde{u} \in C^{1}(\bar{\Omega})$, $0<u_{0} \leq \tilde{u}$ in $\bar{\Omega}, u_{0} \neq \tilde{u}$; 
(b) if $\lambda=\hat{\lambda}$, then problem $\left(P_{\hat{\lambda}}\right)$ has at least one positive solution $\hat{u} \in C^{1}(\bar{\Omega})$;

(c) if $\lambda>\hat{\lambda}$, then problem $\left(P_{\lambda}\right)$ has no positive solution.

The proof of Theorem 1.3 is given in Sections 2 and 3.

2. Lower and upper solution techniques. As a preliminary step in the proof of the theorem, we develop lower and upper solution techniques for problem $\left(P_{\lambda}\right)$.

Let $A: W^{1, p}(\Omega) \rightarrow W^{1, p}(\Omega)^{*}$ be the nonlinear map defined by

$$
\langle A(u), y\rangle=\int_{\Omega}|\nabla u|^{p-2}(\nabla u, \nabla y)_{\boldsymbol{R}^{N}} d x \quad \text { for all } u, y \in W^{1, p}(\Omega) .
$$

It is well-known that this operator is continuous, monotone, and of type $(S)_{+}$(i.e., for every sequence $\left\{u_{k}\right\}_{k \geq 1} \subset W^{1, p}(\Omega)$ such that $u_{k} \stackrel{\mathrm{w}}{\rightarrow} u$ in $W^{1, p}(\Omega)$ and $\lim \sup _{k \rightarrow \infty}\left\langle A\left(u_{k}\right), u_{k}-\right.$ $u\rangle \leq 0$, then $u_{k} \rightarrow u$ in $\left.W^{1, p}(\Omega)\right)$.

Recall that $u \in W^{1, p}(\Omega)$ is called a lower (resp. upper) solution of problem $\left(P_{\lambda}\right)$ if, for each function $v \in W^{1, p}(\Omega), v \geq 0$, we have that

$$
\langle A(u), v\rangle+\int_{\Omega} \beta(x)|u|^{p-2} u v d x-\int_{\Omega} f(x, u(x), \lambda) v d x
$$

is $\leq 0$ (resp. $\geq 0$ ).

We first state a lower and upper solution principle, which will be useful in the sequel. Let $0<\lambda_{1}<\lambda<\lambda_{2}$ and let $u_{1}, u_{2} \in C^{1}(\bar{\Omega})$ be respectively a lower solution of $\left(P_{\lambda_{1}}\right)$ and an upper solution of $\left(P_{\lambda_{2}}\right)$, such that $0<u_{1} \leq u_{2}$ in $\bar{\Omega}$. Consider the following truncation of $f(x, \cdot, \lambda)$ :

$$
\hat{f}(x, s, \lambda)= \begin{cases}f\left(x, u_{1}(x), \lambda\right) & \text { if } s<u_{1}(x) \\ f(x, s, \lambda) & \text { if } u_{1}(x) \leq s \leq u_{2}(x) \\ f\left(x, u_{2}(x), \lambda\right) & \text { if } s>u_{2}(x)\end{cases}
$$

This is a Carathéodory function. We set $\hat{F}(x, s, \lambda)=\int_{0}^{s} \hat{f}(x, t, \lambda) d t$ and introduce the $C^{1}$ functional $\hat{\varphi}_{\lambda}: W^{1, p}(\Omega) \rightarrow \boldsymbol{R}$ defined by

$$
\hat{\varphi}_{\lambda}(u)=\frac{1}{p}\|\nabla u\|_{p}^{p}+\frac{1}{p} \int_{\Omega} \beta|u|^{p} d x-\int_{\Omega} \hat{F}(x, u, \lambda) d x \quad \text { for all } u \in W^{1, p}(\Omega) .
$$

LEMMA 2.1. Let $\lambda, \lambda_{1}, \lambda_{2}, u_{1}, u_{2}, \hat{\varphi}_{\lambda}$ be as above. Assume that $\left(\mathrm{H}_{f}\right)$ and $\left(\mathrm{H}_{\beta}\right)$ hold. Then, there is $u_{0} \in C^{1}(\bar{\Omega})$ a (weak) solution of problem $\left(P_{\lambda}\right)$ which is a global minimizer of $\hat{\varphi}_{\lambda}$, and that satisfies $u_{1}<u_{0}<u_{2}$ in $\bar{\Omega}$.

Proof. As noted in [1, Lemma 2], hypothesis $\left(\mathrm{H}_{\beta}\right)$ implies that there exists a constant $\hat{c}>0$ such that

$$
\|\nabla u\|_{p}^{p}+\int_{\Omega} \beta|u|^{p} d x \geq \hat{c}\|u\|^{p} \quad \text { for all } u \in W^{1, p}(\Omega) .
$$

It is clear from (2) and (3) that $\hat{\varphi}_{\lambda}$ is coercive. Also, exploiting the compact embedding of $W^{1, p}(\Omega)$ into $L^{p}(\Omega)$, we have that $\hat{\varphi}_{\lambda}$ is sequentially weakly lower semicontinuous. So, we 
can find $u_{0} \in W^{1, p}(\Omega)$ such that

$$
\hat{\varphi}_{\lambda}\left(u_{0}\right)=\inf \left\{\hat{\varphi}_{\lambda}(u) ; u \in W^{1, p}(\Omega)\right\} .
$$

From (4) we have $\hat{\varphi}_{\lambda}^{\prime}\left(u_{0}\right)=0$, which reads as

$$
A\left(u_{0}\right)+\beta\left|u_{0}\right|^{p-2} u_{0}=\hat{f}\left(\cdot, u_{0}(\cdot), \lambda\right) .
$$

Acting on (5) with $\left(u_{0}-u_{2}\right)^{+} \in W^{1, p}(\Omega)$, we infer that

$$
\begin{aligned}
& \left\langle A\left(u_{0}\right),\left(u_{0}-u_{2}\right)^{+}\right\rangle+\int_{\Omega} \beta\left|u_{0}\right|^{p-2} u_{0}\left(u_{0}-u_{2}\right)^{+} d x \\
& \quad=\int_{\Omega} \hat{f}\left(x, u_{0}, \lambda\right)\left(u_{0}-u_{2}\right)^{+} d x=\int_{\Omega} f\left(x, u_{2}, \lambda\right)\left(u_{0}-u_{2}\right)^{+} d x \\
& \quad \leq \int_{\Omega} f\left(x, u_{2}, \lambda_{2}\right)\left(u_{0}-u_{2}\right)^{+} d x \\
& \quad \leq\left\langle A\left(u_{2}\right),\left(u_{0}-u_{2}\right)^{+}\right\rangle+\int_{\Omega} \beta u_{2}^{p-1}\left(u_{0}-u_{2}\right)^{+} d x,
\end{aligned}
$$

where we have used (2), $\left(\mathrm{H}_{f}\right)$ (vii) and the fact that $u_{2}$ is an upper solution of $\left(P_{\lambda_{2}}\right)$. So, using the monotonicity of $\xi \mapsto|\xi|^{p-2} \xi$ on $\boldsymbol{R}^{N}$, we infer that

$$
\int_{\left\{u_{0}>u_{2}\right\}} \beta\left(\left|u_{0}\right|^{p-2} u_{0}-\left|u_{2}\right|^{p-2} u_{2}\right)\left(u_{0}-u_{2}\right) d x \leq 0 .
$$

Then, using the strict monotonicity of $s \mapsto|s|^{p-2} s$ on $\boldsymbol{R}$, we obtain that $\left|\left\{u_{0}>u_{2}\right\}\right|_{N}=0$, i.e., $u_{0} \leq u_{2}$. Similarly, acting on (5) with $\left(u_{0}-u_{1}\right)^{-}$yields $u_{1} \leq u_{0}$. It follows that $u_{0} \in\left[u_{1}, u_{2}\right]:=\left\{u \in W^{1, p}(\Omega) ; u_{1}(x) \leq u(x) \leq u_{2}(x)\right.$ for a.a. $\left.x \in \Omega\right\}$. Then, by virtue of (2), equation (5) becomes

$$
A\left(u_{0}\right)+\beta u_{0}^{p-1}=f\left(\cdot, u_{0}(\cdot), \lambda\right) .
$$

Consequently, $u_{0}$ is a (nontrivial) solution of problem $\left(P_{\lambda}\right)$ (see [12]). Nonlinear regularity theory (see [9]) implies that $u_{0} \in C^{1}(\bar{\Omega})$.

It remains to show that $u_{1}<u_{0}<u_{2}$ in $\bar{\Omega}$. We only show that $u_{0}<u_{2}$, the proof of the other inequality being similar. Corresponding to $\lambda_{2}$ and $\rho:=\left\|u_{2}\right\|_{\infty}$, we consider $\sigma_{\rho}=\sigma_{\rho}\left(\lambda_{2}\right)>0$ given in $\left(\mathrm{H}_{f}\right)(\mathrm{vi})$. For $\delta>0$ small, we set $u_{\delta}=u_{0}+\delta$. We can write

$$
\begin{aligned}
-\Delta_{p} & u_{\delta}(x)+\left(\beta(x)+\sigma_{\rho}\right) u_{\delta}(x)^{p-1} \\
& \leq-\Delta_{p} u_{0}(x)+\left(\beta(x)+\sigma_{\rho}\right) u_{0}(x)^{p-1}+\gamma(\delta) \\
& =f\left(x, u_{0}(x), \lambda\right)+\sigma_{\rho} u_{0}(x)^{p-1}+\gamma(\delta) \\
& =f\left(x, u_{0}(x), \lambda_{2}\right)+\sigma_{\rho} u_{0}(x)^{p-1}+f\left(x, u_{0}(x), \lambda\right)-f\left(x, u_{0}(x), \lambda_{2}\right)+\gamma(\delta) \\
& \leq f\left(x, u_{2}(x), \lambda_{2}\right)+\sigma_{\rho} u_{2}(x)^{p-1}-\theta_{0}\left(t, \lambda_{2}, \lambda\right)+\gamma(\delta),
\end{aligned}
$$

with $\gamma(\delta) \rightarrow 0$ as $\delta \downarrow 0$, and $t:=\min _{\bar{\Omega}} u_{0}>0$, where we have used $\left(\mathrm{H}_{f}\right)$ (vi), (vii) and that $u_{0} \leq u_{2}$. Since $\gamma(\delta) \rightarrow 0$ as $\delta \downarrow 0$ and $\theta_{0}\left(t, \lambda_{2}, \lambda\right)>0\left(\right.$ see $\left(\mathrm{H}_{f}\right)($ vii) $)$, for $\delta \in(0,1)$ small we have

$$
-\Delta_{p} u_{\delta}(x)+\left(\beta(x)+\sigma_{\rho}\right) u_{\delta}(x)^{p-1} \leq-\Delta_{p} u_{2}(x)+\left(\beta(x)+\sigma_{\rho}\right) u_{2}(x)^{p-1} \text { in } W^{1, p}(\Omega)^{*} .
$$


Acting on the previous inequality with the test function $\left(u_{2}-u_{\delta}\right)^{-} \in W^{1, p}(\Omega)$ yields $u_{\delta} \leq$ $u_{2}$. Thus $u_{0}<u_{2}$.

Our next purpose is to show existence results for lower and upper solutions for the problem $\left(P_{\lambda}\right)$.

Lemma 2.2. Assume that $\left(\mathrm{H}_{f}\right)$ and $\left(\mathrm{H}_{\beta}\right)$ hold. Then, for every $\lambda>0$, any small enough constant $\delta>0$ is a lower solution of problem $\left(P_{\lambda}\right)$.

Proof. Let $q=q(\lambda) \in[1, p)$ and $\eta_{0}=\eta_{0}(\lambda)>0$ be as in $\left(\mathrm{H}_{f}\right)(\mathrm{v})$. Let $\delta \in$ $\left(0,\left(\eta_{0} /\|\beta\|_{\infty}\right)^{1 /(p-q)}\right]\left(\mathrm{cf} .\left(\mathrm{H}_{\beta}\right)\right)$. Then, we see that

$$
\int_{\Omega} \beta(x) \delta^{p-1} v d x-\int_{\Omega} f(x, \delta, \lambda) v d x \leq\left(\|\beta\|_{\infty} \delta^{p-1}-\eta_{0} \delta^{q-1}\right)\|v\|_{1} \leq 0
$$

for all $v \in W^{1, p}(\Omega), v \geq 0$, which yields that $\delta$ is a (constant) lower solution of problem $\left(P_{\lambda}\right)$.

Lemma 2.3. Assume that $\left(\mathrm{H}_{f}\right)$ and $\left(\mathrm{H}_{\beta}\right)$ hold. Then, there exists $\lambda_{0}>0$ such that for every $\lambda \in\left(0, \lambda_{0}\right)$, problem $\left(P_{\lambda}\right)$ admits an upper solution $\bar{u} \in C^{1}(\bar{\Omega}), \bar{u}>0$ in $\bar{\Omega}$.

PROOF. The proof comprises three steps.

Step 1: the auxiliary nonlinear Neumann problem

$$
\begin{cases}-\Delta_{p} e(x)+\beta(x)|e(x)|^{p-2} e(x)=1 & \text { in } \Omega, \\ \frac{\partial e}{\partial n_{p}}=0 & \text { on } \partial \Omega\end{cases}
$$

has a unique solution $e \in W^{1, p}(\Omega)$, moreover $e \in C^{1}(\bar{\Omega}), \min _{\bar{\Omega}} e \geq\left(1 /\|\beta\|_{\infty}\right)^{1 /(p-1)}$.

The Euler functional associated to problem (6) is coercive and strictly convex, thus (6) admits a unique solution $e \in W^{1, p}(\Omega)$. Nonlinear regularity theory ensures that $e \in C^{1}(\bar{\Omega})$. Acting on (6) with $-e^{-}$, we deduce that $e \geq 0$. Finally, acting on (6) with the test function $-(e-\mu)^{-}$, with $\mu=\left(1 /\|\beta\|_{\infty}\right)^{1 /(p-1)}$, yields the relation

$$
\left\|\nabla(e-\mu)^{-}\right\|_{p}^{p}=\int_{\{e<\mu\}}(\mu-e)\left(\beta(x) e(x)^{p-1}-1\right) d x \leq 0,
$$

whence $e \geq \mu$ in $\Omega$. This establishes Step 1 .

Step 2: there exists $\lambda_{0}>0$ such that for every $\lambda \in\left(0, \lambda_{0}\right)$ there is $\tau(\lambda)>0$ with

$$
a(\lambda)+c\left(\tau(\lambda)\|e\|_{\infty}\right)^{r(\lambda)-1}<\tau(\lambda)^{p-1},
$$

where $a(\lambda), r(\lambda)$, and $c>0$ are given by $\left(\mathrm{H}_{f}\right)(\mathrm{i})$.

Arguing by contradiction, suppose that this is not true. Let $r_{-}=\inf _{\lambda \in(0,+\infty)} r(\lambda)$ and $r_{+}=\sup _{\lambda \in(0,+\infty)} r(\lambda)$. Then we can find a sequence $\left\{\lambda_{k}\right\}_{k \geq 1} \subset(0,+\infty)$ such that $\lambda_{k} \downarrow 0$ as $k \rightarrow \infty$ and

$$
\begin{aligned}
\tau^{p-1} & \leq a\left(\lambda_{k}\right)+c\left(\tau\|e\|_{\infty}\right)^{r\left(\lambda_{k}\right)-1} \\
& \leq a\left(\lambda_{k}\right)+c\left(\tau\|e\|_{\infty}\right)^{r_{-}-1}+c\left(\tau\|e\|_{\infty}\right)^{r_{+}-1} \text { for all } \tau>0, k \geq 1,
\end{aligned}
$$


which implies that $1 \leq c \tau^{r_{-}-p}\|e\|_{\infty}^{r_{-}-1}+c \tau^{r_{+}-p}\|e\|_{\infty}^{r_{+}-1}$. Since $r_{-}, r_{+}>p\left(\operatorname{see}\left(\mathrm{H}_{f}\right)(\mathrm{i})\right)$ and $\tau>0$ is arbitrary, we let $\tau \downarrow 0$ to reach a contradiction. This proves Step 2 .

Step 3: given $\lambda \in\left(0, \lambda_{0}\right)$ and letting $\tau=\tau(\lambda)$ be as in Step 2, the function $\bar{u}=\tau e$ is an upper solution of problem $\left(P_{\lambda}\right)$, and moreover $\bar{u} \in C^{1}(\bar{\Omega})$ and $\bar{u}>0$.

Using (6), $\left(\mathrm{H}_{f}\right)(\mathrm{i})$ and (7), we note that

$$
\langle A(\bar{u}), v\rangle+\int_{\Omega} \beta(x) \bar{u}^{p-1} v d x=\int_{\Omega} \tau^{p-1} v d x \geq \int_{\Omega} f(x, \bar{u}(x), \lambda) v d x
$$

for all $v \in W^{1, p}(\Omega), v \geq 0$. So, $\bar{u}$ is an upper solution of problem $\left(P_{\lambda}\right)$.

\section{Proof of Theorem 1.3. We set}

$$
\mathcal{S}=\left\{\lambda>0 ;\left(P_{\lambda}\right) \text { admits a positive solution }\right\}
$$

The first step of the proof of Theorem 1.3 is to show the nonemptiness of $\mathcal{S}$.

Proposition 3.1. Assume that $\left(\mathrm{H}_{f}\right)$ and $\left(\mathrm{H}_{\beta}\right)$ hold. Then $\mathcal{S} \neq \emptyset$ and if $\lambda \in \mathcal{S}$ and $\mu \in(0, \lambda)$, then $\mu \in \mathcal{S}$.

ProOF. By Lemma 2.3 , there is $\bar{\lambda}>0$ such that problem $\left(P_{\bar{\lambda}}\right)$ admits an upper solution $\bar{u} \in C^{1}(\bar{\Omega}), \bar{u}>0$. In order to establish the proposition, it is sufficient to show that, if $\bar{\lambda}>0$ is such that $\left(P_{\bar{\lambda}}\right)$ admits such an upper solution, then for every $\lambda \in(0, \bar{\lambda})$, we have $\lambda \in \mathcal{S}$. Fix $\lambda_{1}<\lambda$. By Lemma 2.2 , choosing $\delta \in\left(0, \min _{\bar{\Omega}} \bar{u}\right)$ small enough, $u_{1}:=\delta$ is a lower solution of $\left(P_{\lambda_{1}}\right)$. Then, Lemma 2.1 ensures that problem $\left(P_{\lambda}\right)$ admits a solution $u_{0} \in C^{1}(\bar{\Omega}), u_{0}>0$. Thereby, $\lambda \in \mathcal{S}$.

We let $\hat{\lambda}=\sup \mathcal{S}$. The previous proposition shows that $\hat{\lambda}>0$.

Proposition 3.2. If hypotheses $\left(\mathrm{H}_{f}\right)$ and $\left(\mathrm{H}_{\beta}\right)$ hold, then $\hat{\lambda}<+\infty$.

Proof. Fix $\mu \geq\|\beta\|_{\infty}$. We claim that there exists $\tilde{\lambda}>0$ such that

$$
f(x, s, \tilde{\lambda}) \geq \mu s^{p-1} \quad \text { for a.a. } x \in \Omega \text {, all } s \geq 0 .
$$

Fixing $\lambda_{0}>0$, by $\left(\mathrm{H}_{f}\right)(\mathrm{ii}),(\mathrm{v})$, we find $s_{0}>0$ and $\varepsilon:=\min \left\{\left(\eta_{0} / \mu\right)^{1 /(p-q)}, s_{0}\right\}>0$ such that

$$
\begin{gathered}
f\left(x, s, \lambda_{0}\right) \geq \mu s^{p-1} \quad \text { for a.a. } x \in \Omega, \text { all } s>s_{0}, \\
f\left(x, s, \lambda_{0}\right) \geq \eta \eta_{0} s^{q-1} \geq \mu s^{p-1} \quad \text { for a.a. } x \in \Omega \text {, all } s \in(0, \varepsilon),
\end{gathered}
$$

where $q=q\left(\lambda_{0}\right) \in[1, p)$ and $\eta_{0}=\eta_{0}\left(\lambda_{0}\right)>0$ are as in $\left(\mathrm{H}_{f}\right)(\mathrm{v})$. Since $\theta(\varepsilon, \lambda) \rightarrow+\infty$ as $\lambda \rightarrow+\infty\left(\operatorname{see}\left(\mathrm{H}_{f}\right)(\mathrm{iv})\right)$, we can find $\tilde{\lambda}>\lambda_{0}$ such that $\theta(\varepsilon, \tilde{\lambda}) \geq \mu s_{0}^{p-1}$. By $\left(\mathrm{H}_{f}\right)(\mathrm{iv})$, we have

$$
f(x, s, \tilde{\lambda}) \geq \theta(\varepsilon, \tilde{\lambda}) \geq \mu s_{0}^{p-1} \geq \mu s^{p-1} \quad \text { for a.a. } x \in \Omega, \text { all } s \in\left[\varepsilon, s_{0}\right] .
$$

Because $f(x, s, \cdot)$ is nondecreasing (see $\left(\mathrm{H}_{f}\right)$ (vii)), altogether we deduce formula (8) and this shows our claim. 
Let $\lambda>\tilde{\lambda}$ and suppose that $\lambda \in \mathcal{S}$. Then there exists $u_{\lambda} \in C^{1}(\bar{\Omega})$ positive solution of $\left(P_{\lambda}\right)$. Let $t=\min _{\bar{\Omega}} u_{\lambda}$. For $\delta>0$, we set $t_{\delta}=t+\delta$. Corresponding to $\rho:=\left\|u_{\lambda}\right\|_{\infty}$, we consider $\sigma_{\rho}=\sigma_{\rho}(\lambda)>0$ given in $\left(\mathrm{H}_{f}\right)(\mathrm{vi})$. By (8), and using $\left(\mathrm{H}_{f}\right)$ (vii), we can write

$$
\begin{aligned}
-\Delta_{p} t_{\delta}+\left(\beta(x)+\sigma_{\rho}\right) t_{\delta}^{p-1} & \leq\left(\mu+\sigma_{\rho}\right) t^{p-1}+\gamma(\delta) \leq f(x, t, \tilde{\lambda})+\sigma_{\rho} t^{p-1}+\gamma(\delta) \\
& =f(x, t, \lambda)+\sigma_{\rho} t^{p-1}+f(x, t, \tilde{\lambda})-f(x, t, \lambda)+\gamma(\delta) \\
& \leq f\left(x, u_{\lambda}(x), \lambda\right)+\sigma_{\rho} u_{\lambda}(x)^{p-1}-\theta_{0}(t, \lambda, \tilde{\lambda})+\gamma(\delta),
\end{aligned}
$$

with $\gamma(\delta) \rightarrow 0$ as $\delta \downarrow 0$. Since $\gamma(\delta) \rightarrow 0$ as $\delta \downarrow 0$ and $\theta_{0}(t, \lambda, \tilde{\lambda})>0$ (see $\left(\mathrm{H}_{f}\right)$ (vii)), for $\delta>0$ small we have

$$
-\Delta_{p} t_{\delta}+\left(\beta(x)+\sigma_{\rho}\right) t_{\delta}^{p-1} \leq-\Delta_{p} u_{\lambda}(x)+\left(\beta(x)+\sigma_{\rho}\right) u_{\lambda}(x)^{p-1} \text { in } W^{1, p}(\Omega)^{*} .
$$

This implies that $t_{\delta} \leq u_{\lambda}$, which is a contradiction since $t$ is the minimum of $u_{\lambda}$. Therefore, $\lambda \notin \mathcal{S}$, and so $\hat{\lambda} \leq \tilde{\lambda}<+\infty$.

Proposition 3.3. If hypotheses $\left(\mathrm{H}_{f}\right)$ and $\left(\mathrm{H}_{\beta}\right)$ hold, then $\hat{\lambda} \in \mathcal{S}$, and so $\mathcal{S}=(0, \hat{\lambda}]$.

Proof. Let $\left\{\lambda_{k}\right\}_{k \geq 1} \subset \mathcal{S}$ be such that $\lambda_{k} \uparrow \hat{\lambda}$ as $k \rightarrow \infty$. Let $u_{k}:=u_{\lambda_{k}} \in C^{1}(\bar{\Omega})$ be a positive solution for problem $\left(P_{\lambda_{k}}\right), k \geq 1$. Let $\varphi_{\lambda_{k}}: W^{1, p}(\Omega) \rightarrow \boldsymbol{R}$ be the $C^{1}$-functional for problem $\left(P_{\lambda_{k}}\right)$ defined by

$$
\varphi_{\lambda_{k}}(u)=\frac{1}{p}\|\nabla u\|_{p}^{p}+\frac{1}{p} \int_{\Omega} \beta|u|^{p} d x-\int_{\Omega} F\left(x, u, \lambda_{k}\right) d x \quad \text { for all } u \in W^{1, p}(\Omega) .
$$

Let us show that, without any loss of generality, we may assume that

$$
\varphi_{\lambda_{k}}\left(u_{k}\right)<0 \text { for all } k \geq 1 \text {. }
$$

To do this, fix $k \geq 1$. By Lemma 2.2 , choosing $\delta_{k} \in\left(0, \min _{\bar{\Omega}} u_{k+1}\right)$ small enough, we have that the constant function $\delta_{k}$ is a lower solution of problem $\left(P_{\lambda_{k} / 2}\right)$. By $\left(\mathrm{H}_{f}\right)(\mathrm{v})$, choosing $\delta_{k}>0$ even smaller if necessary, we may assume that $\varphi_{\lambda_{k}}\left(\delta_{k}\right)<0$. Applying Lemma 2.1 for the parameters $\lambda_{k} / 2<\lambda_{k}<\lambda_{k+1}$, the lower solution $\delta_{k}$ of $\left(P_{\lambda_{k} / 2}\right)$ and the upper solution $u_{k+1}$ of $\left(P_{\lambda_{k+1}}\right)$, we find $\tilde{u}_{k} \in C^{1}(\bar{\Omega})$ positive solution of $\left(P_{\lambda_{k}}\right)$ that satisfies $\delta_{k}<\tilde{u}_{k}<u_{k+1}$ in $\bar{\Omega}$ and $\varphi_{\lambda_{k}}\left(\tilde{u}_{k}\right) \leq \varphi_{\lambda_{k}}\left(\delta_{k}\right)<0$. Up to dealing with $\tilde{u}_{k}$ instead of $u_{k}$, we indeed may assume that (9) holds.

We have

$$
A\left(u_{k}\right)+\beta u_{k}^{p-1}=f\left(\cdot, u_{k}(\cdot), \lambda_{k}\right) \text { for all } k \geq 1 .
$$

Acting on (10) with $u_{k}$, we obtain

$$
-\left\|\nabla u_{k}\right\|_{p}^{p}-\int_{\Omega} \beta u_{k}^{p} d x+\int_{\Omega} f\left(x, u_{k}, \lambda_{k}\right) u_{k} d x=0 \quad \text { for all } k \geq 1 .
$$

On the other hand, from (9) we have

$$
\left\|\nabla u_{k}\right\|_{p}^{p}+\int_{\Omega} \beta u_{k}^{p} d x-\int_{\Omega} p F\left(x, u_{k}, \lambda_{k}\right) d x<0 \text { for all } k \geq 1 .
$$


Adding (11) and (12) results in

$$
\int_{\Omega} \xi_{\lambda_{k}}\left(x, u_{k}\right) d x<0 \text { for all } k \geq 1
$$

(see the notation in $\left(\mathrm{H}_{f}\right)$ (iii)).

CLAIM. $\quad\left\{u_{k}\right\}_{k \geq 1}$ is bounded in $W^{1, p}(\Omega)$.

Arguing by contradiction, suppose that the Claim is not true. Then, by passing to a suitable subsequence if necessary, we may assume that $\left\|u_{k}\right\| \rightarrow+\infty$. Let $y_{k}=u_{k} /\left\|u_{k}\right\|$, $k \geq 1$. Then $\left\|y_{k}\right\|=1, y_{k}>0$ for all $k \geq 1$, and so we may assume that

$y_{k} \stackrel{\mathrm{w}}{\rightarrow} y$ in $W^{1, p}(\Omega), y_{k} \rightarrow y$ in $L^{r}(\Omega)$ for all $r \in\left[1, p^{*}\right), y_{k}(x) \rightarrow y(x)$ for a.a. $x \in \Omega$, with $y \geq 0$.

Case 1: $y \neq 0$.

Let $Z(y)=\{x \in \Omega ; y(x)=0\}$. Since $y \neq 0$, we have that $|\Omega \backslash Z(y)|_{N}>0$, and so $u_{k}(x) \rightarrow+\infty$ for a.a. $x \in \Omega \backslash Z(y)$. Recalling that $\lambda_{k} \geq \lambda_{1}, k \geq 1$, by virtue of $\left(\mathrm{H}_{f}\right)(\mathrm{vii})$, we have

$$
F\left(x, u_{k}(x), \lambda_{k}\right) \geq F\left(x, u_{k}(x), \lambda_{1}\right) \quad \text { for a.a. } x \in \Omega, \text { all } k \geq 1 .
$$

Moreover, $\left(\mathrm{H}_{f}\right)$ (ii) implies that

$$
\frac{F\left(x, u_{k}(x), \lambda_{1}\right)}{\left\|u_{k}\right\|^{p}}=\frac{F\left(x, u_{k}(x), \lambda_{1}\right)}{u_{k}(x)^{p}} y_{k}(x)^{p} \rightarrow+\infty \quad \text { for a.a. } x \in \Omega \backslash Z(y) .
$$

Through Fatou's lemma (since $F\left(x, u_{k}(x), \lambda_{1}\right) \geq 0$ by $\left.\left(\mathrm{H}_{f}\right)(\mathrm{v})\right)$, we deduce that

$$
\int_{\Omega} \frac{F\left(x, u_{k}(x), \lambda_{1}\right)}{\left\|u_{k}\right\|^{p}} d x \rightarrow+\infty \quad \text { as } k \rightarrow \infty .
$$

Then (15) leads to

$$
\int_{\Omega} \frac{F\left(x, u_{k}(x), \lambda_{k}\right)}{\left\|u_{k}\right\|^{p}} d x \rightarrow+\infty \text { as } k \rightarrow \infty .
$$

Note by $\left(\mathrm{H}_{f}\right)$ (iii) that

$$
f\left(x, u_{k}, \lambda_{k}\right) u_{k}-p F\left(x, u_{k}, \lambda_{k}\right) \geq-\beta_{\lambda_{k}}^{*}(x) \text { for all } k \geq 1 .
$$

Using (10), the fact that $\left\|y_{k}\right\|=1$, and the boundedness of $\left\{\left\|\beta_{\lambda_{k}}^{*}\right\|_{1}\right\}_{k \geq 1}$ as known from $\left(\mathrm{H}_{f}\right)$ (iii), we obtain that

$$
\begin{aligned}
\int_{\Omega} \frac{p F\left(x, u_{k}, \lambda_{k}\right)}{\left\|u_{k}\right\|^{p}} d x & \leq \int_{\Omega} \frac{f\left(x, u_{k}, \lambda_{k}\right) u_{k}+\beta_{\lambda_{k}}^{*}(x)}{\left\|u_{k}\right\|^{p}} d x \\
& =\left\|\nabla y_{k}\right\|_{p}^{p}+\int_{\Omega} \beta y_{k}^{p} d x+\frac{\left\|\beta_{\lambda_{k}}^{*}\right\|_{1}}{\left\|u_{k}\right\|^{p}} \leq M_{1} \quad \text { for all } k \geq 1,
\end{aligned}
$$

for some $M_{1}>0$. This contradicts (16).

Case 2: $y=0$. 
For every $k \geq 1$, let $t_{k} \in[0,1]$ be such that

$$
\varphi_{\lambda_{k}}\left(t_{k} u_{k}\right)=\max \left\{\varphi_{\lambda_{k}}\left(t u_{k}\right) ; t \in[0,1]\right\} .
$$

Fix $\gamma>0$ and set $v_{k}=(2 \gamma p / \hat{c})^{1 / p} y_{k}, k \geq 1$ (with $\hat{c}>0$ as in (3)). By $\left(\mathrm{H}_{f}\right)(\mathrm{i}),(\mathrm{v}),(\mathrm{vii})$, we have

$$
0 \leq F\left(x, v_{k}(x), \lambda_{k}\right) \leq F\left(x, v_{k}(x), \hat{\lambda}\right) \leq a(\hat{\lambda}) v_{k}(x)+\frac{c}{r(\hat{\lambda})} v_{k}(x)^{r(\hat{\lambda})}
$$

for a.a. $x \in \Omega$. According to (14), $v_{k} \rightarrow 0$ in $L^{r(\hat{\lambda})}(\Omega)$ as $k \rightarrow \infty$, hence we obtain

$$
\int_{\Omega} F\left(x, v_{k}(x), \lambda_{k}\right) d x \rightarrow 0 \text { as } k \rightarrow \infty .
$$

Since $\left\|u_{k}\right\| \rightarrow+\infty$, we can find an integer $k_{0} \geq 1$ such that $(2 \gamma p / \hat{c})^{1 / p} /\left\|u_{k}\right\| \in(0,1)$ for all $k \geq k_{0}$. By (17), (3), the fact that $\left\|y_{k}\right\|=1$ and (18), this yields

$$
\begin{aligned}
\varphi_{\lambda_{k}}\left(t_{k} u_{k}\right) & \geq \varphi_{\lambda_{k}}\left(v_{k}\right)=\frac{1}{p}\left\|\nabla v_{k}\right\|_{p}^{p}+\frac{1}{p} \int_{\Omega} \beta v_{k}^{p} d x-\int_{\Omega} F\left(x, v_{k}(x), \lambda_{k}\right) d x \\
& \geq \frac{\hat{c}}{p}\left\|v_{k}\right\|^{p}-\int_{\Omega} F\left(x, v_{k}(x), \lambda_{k}\right) d x \\
& =2 \gamma-\int_{\Omega} F\left(x, v_{k}(x), \lambda_{k}\right) d x \geq \gamma \quad \text { for all } k \geq k_{1},
\end{aligned}
$$

for some $k_{1} \geq k_{0}$. Recalling that $\gamma>0$ is arbitrary, we see that

$$
\varphi_{\lambda_{k}}\left(t_{k} u_{k}\right) \rightarrow+\infty \text { as } k \rightarrow \infty \text {. }
$$

Since $0 \leq t_{k} u_{k} \leq u_{k}$, in view of $\left(\mathrm{H}_{f}\right)$ (iii), we have

$$
\int_{\Omega} \xi_{\lambda_{k}}\left(x, t_{k} u_{k}\right) d x \leq \int_{\Omega} \xi_{\lambda_{k}}\left(x, u_{k}\right) d x+\left\|\beta_{\lambda_{k}}^{*}\right\|_{1} .
$$

Note that $\varphi_{\lambda_{k}}\left(u_{k}\right)<0=\varphi_{\lambda_{k}}(0)$ (see (9)), hence $t_{k} \neq 1$ for all $k \geq 1$, according to (17). Moreover, the fact that $\varphi_{\lambda_{k}}(0)=0$ and (19) imply that $t_{k} \in(0,1)$ for all $k \geq k_{2}$, with $k_{2}$ large enough. We infer that

$$
\begin{aligned}
0 & =\left.t_{k} \frac{d}{d t} \varphi_{\lambda_{k}}\left(t u_{k}\right)\right|_{t=t_{k}}=\left\langle\varphi_{\lambda_{k}}^{\prime}\left(t_{k} u_{k}\right), t_{k} u_{k}\right\rangle \\
& =\left\|\nabla\left(t_{k} u_{k}\right)\right\|_{p}^{p}+\int_{\Omega} \beta\left(t_{k} u_{k}\right)^{p} d x-\int_{\Omega} f\left(x, t_{k} u_{k}(x), \lambda_{k}\right) t_{k} u_{k} d x
\end{aligned}
$$

for all $k \geq k_{2}$. Using (21), (20) and $\left(\mathrm{H}_{f}\right)$ (iii), we obtain

$$
p \varphi_{\lambda_{k}}\left(t_{k} u_{k}\right) \leq \int_{\Omega} \xi_{\lambda_{k}}\left(x, u_{k}\right) d x+\left\|\beta_{\lambda_{k}}^{*}\right\|_{1} \leq \int_{\Omega} \xi_{\lambda_{k}}\left(x, u_{k}\right) d x+M_{2}
$$

for all $k \geq k_{2}$, with some $M_{2}>0$. Comparing (13), (19) and (22), we arrive at a contradiction. This proves the Claim.

By virtue of the Claim, we may assume that

$$
u_{k} \stackrel{\mathrm{w}}{\rightarrow} \hat{u} \text { in } W^{1, p}(\Omega), u_{k} \rightarrow \hat{u} \text { in } L^{r}(\Omega) \text { for all } r \in\left[1, p^{*}\right), u_{k}(x) \rightarrow \hat{u}(x) \text { for a.a. } x \in \Omega,
$$


with $\hat{u} \geq 0$. Due to (10), $\left(\mathrm{H}_{f}\right)$ (i), (v), (vii), we have

$$
\begin{aligned}
& \left\langle A\left(u_{k}\right), u_{k}-\hat{u}\right\rangle+\int_{\Omega} \beta u_{k}^{p-1}\left(u_{k}-\hat{u}\right) d x \\
& \quad=\int_{\Omega} f\left(x, u_{k}, \lambda_{k}\right)\left(u_{k}-\hat{u}\right) d x \leq \int_{\Omega} f\left(x, u_{k}, \hat{\lambda}\right)\left|u_{k}-\hat{u}\right| d x \\
& \quad \leq \int_{\Omega}\left(a(\hat{\lambda})\left|u_{k}-\hat{u}\right|+c u_{k}^{r(\hat{\lambda})-1}\left|u_{k}-\hat{u}\right|\right) d x .
\end{aligned}
$$

Passing to the limit as $k \rightarrow \infty$ and using (23), we get $\lim _{\sup } \sup _{k \rightarrow \infty}\left\langle A\left(u_{k}\right), u_{k}-\hat{u}\right\rangle \leq 0$, which implies that $u_{k} \rightarrow \hat{u}$ in $W^{1, p}(\Omega)$ as $k \rightarrow \infty$ (since $A$ is of type $(S)_{+}$). From (10), using that $f$ is a Carathéodory function, (23), the fact that $\lambda_{k} \uparrow \hat{\lambda}$ as $k \rightarrow \infty$, and $\left(\mathrm{H}_{f}\right)$ (vii), we obtain

$$
A(\hat{u})+\beta|\hat{u}|^{p-2} \hat{u}=f(\cdot, \hat{u}(\cdot), \hat{\lambda}) .
$$

Hence, $\hat{u}$ is a solution of problem $\left(P_{\hat{\lambda}}\right)$ (see [12]). Due to nonlinear regularity theory, we see that $\hat{u} \in C^{1}(\bar{\Omega})$.

It remains to show that $\hat{u}>0$. To this end, let $q=q\left(\lambda_{1}\right) \in[1, p)$ and $\eta_{0}=\eta_{0}\left(\lambda_{1}\right)>0$ be as in $\left(\mathrm{H}_{f}\right)(\mathrm{v})$. Since $\lambda_{k} \geq \lambda_{1}$ for all $k \geq 1$, and by virtue of $\left(\mathrm{H}_{f}\right)$ (vii), we get

$$
\eta_{0} s^{q-1} \leq f\left(x, s, \lambda_{1}\right) \leq f\left(x, s, \lambda_{k}\right) \quad \text { for a.a. } x \in \Omega \text {, all } s \geq 0 \text {, all } k \geq 1 .
$$

Let us show that $\min _{\bar{\Omega}} u_{k} \geq \delta_{0}:=\left(\eta_{0} /\|\beta\|_{\infty}\right)^{1 /(p-q)}$ for all $k \geq 1$. Arguing by contradiction, suppose that $t_{k}:=\left(1 / \delta_{0}\right) \min _{\bar{\Omega}} u_{k} \in(0,1)$ for some $k \geq 1$. Let $\sigma_{\rho}=\sigma_{\rho}\left(\lambda_{k}\right)>0$, corresponding to $\rho:=\left\|u_{k}\right\|_{\infty}$, be as in $\left(\mathrm{H}_{f}\right)(\mathrm{vi})$. For $\delta>0$, let $t_{\delta, k}=t_{k} \delta_{0}+\delta$. It turns out that

$$
\begin{aligned}
& \left(\beta(x)+\sigma_{\rho}\right) t_{\delta, k}^{p-1} \leq\left(\beta(x)+\sigma_{\rho}\right)\left(t_{k} \delta_{0}\right)^{p-1}+\gamma(\delta) \\
& \leq t_{k}^{p-1} \eta_{0} \delta_{0}^{q-1}+\sigma_{\rho}\left(t_{k} \delta_{0}\right)^{p-1}+\gamma(\delta) \\
& =\eta_{0}\left(t_{k} \delta_{0}\right)^{q-1}+\sigma_{\rho}\left(t_{k} \delta_{0}\right)^{p-1}+\left(t_{k}^{p-1}-t_{k}^{q-1}\right) \eta_{0} \delta_{0}^{q-1}+\gamma(\delta),
\end{aligned}
$$

with $\gamma(\delta) \rightarrow 0$ as $\delta \downarrow 0$. Since $t_{k} \in(0,1)$ and $q<p$, we have $t_{k}^{p-1}-t_{k}^{q-1}<0$, hence for $\delta>0$ small there holds $\left(t_{k}^{p-1}-t_{k}^{q-1}\right) \eta_{0} \delta_{0}^{q-1}+\gamma(\delta) \leq 0$, and so

$$
\begin{aligned}
-\Delta_{p} t_{\delta, k}+\left(\beta(x)+\sigma_{\rho}\right) t_{\delta, k}^{p-1} & \leq \eta_{0}\left(t_{k} \delta_{0}\right)^{q-1}+\sigma_{\rho}\left(t_{k} \delta_{0}\right)^{p-1} \\
& \leq f\left(x, t_{k} \delta_{0}, \lambda_{k}\right)+\sigma_{\rho}\left(t_{k} \delta_{0}\right)^{p-1} \\
& \leq f\left(x, u_{k}(x), \lambda_{k}\right)+\sigma_{\rho} u_{k}(x)^{p-1} \\
& =-\Delta_{p} u_{k}(x)+\left(\beta(x)+\sigma_{\rho}\right) u_{k}(x)^{p-1}
\end{aligned}
$$

in $W^{1, p}(\Omega)^{*}$. Here we used (25), (24) and $\left(\mathrm{H}_{f}\right)$ (vi). It follows that $t_{\delta, k} \leq u_{k}$, and thus $t_{k} \delta_{0}<\min _{\bar{\Omega}} u_{k}$, which contradicts the definition of $t_{k}$. Thus, $t_{k} \geq 1$ for all $k \geq 1$.

Therefore $u_{k} \geq \delta_{0}$ in $\bar{\Omega}$ for all $k \geq 1$. From (23) we derive that $\hat{u} \geq \delta_{0}$ in $\bar{\Omega}$. In particular, $\hat{u}>0$ in $\bar{\Omega}$. Therefore we have $\hat{\lambda} \in \mathcal{S}$, and so $\mathcal{S}=(0, \hat{\lambda}]$. 
Proposition 3.4. If hypotheses $\left(\mathrm{H}_{f}\right)$ and $\left(\mathrm{H}_{\beta}\right)$ hold and $\lambda \in(0, \hat{\lambda})$, then problem $\left(P_{\lambda}\right)$ has at least two positive solutions $u_{0}, \tilde{u} \in C^{1}(\bar{\Omega}), \tilde{u} \geq u_{0}>0$ in $\bar{\Omega}, u_{0} \neq \tilde{u}$ and $u_{0}$ is a local minimizer of the energy functional $\varphi_{\lambda}$ associated to problem $\left(P_{\lambda}\right)$.

Proof. From Proposition 3.3, we know that $\hat{\lambda} \in \mathcal{S}$. Let $\hat{u} \in C^{1}(\bar{\Omega}), \hat{u}>0$, be a solution for problem $\left(P_{\hat{\lambda}}\right)$. By Lemma 2.2 , we find a constant $\delta_{0} \in\left(0, \min _{\bar{\Omega}} \hat{u}\right)$ which is a lower solution of problem $\left(P_{\lambda / 2}\right)$. By Lemma 2.1 applied with $\lambda_{1}=\lambda / 2, \lambda_{2}=\hat{\lambda}, u_{1}=\delta_{0}$ and $u_{2}=\hat{u}$, problem $\left(P_{\lambda}\right)$ admits a solution $u_{0} \in C^{1}(\bar{\Omega})$ with $\delta_{0}<u_{0}<\hat{u}$ in $\bar{\Omega}$, and which is a global minimizer of the corresponding truncated functional $\hat{\varphi}_{\lambda}$. Since the restrictions of $\hat{\varphi}_{\lambda}$ and $\varphi_{\lambda}$ to the ordered interval $\left[\delta_{0}, \hat{u}\right]=\left\{u \in W^{1, p}(\Omega) ; \delta_{0} \leq u(x) \leq \hat{u}(x)\right.$ for a.a. $\left.x \in \Omega\right\}$ coincide, it follows that $u_{0}$ is a local $C^{1}(\bar{\Omega})$-minimizer of $\varphi_{\lambda}$. Invoking [11, Proposition 24], we infer that $u_{0}$ is also a local $W^{1, p}(\Omega)$-minimizer of $\varphi_{\lambda}$.

We consider the following truncation of $f(x, \cdot, \lambda)$ :

$$
g(x, s, \lambda)= \begin{cases}f\left(x, u_{0}(x), \lambda\right) & \text { if } s \leq u_{0}(x) \\ f(x, s, \lambda) & \text { if } s>u_{0}(x) .\end{cases}
$$

This is a Carathéodory function. We set $G(x, s, \lambda)=\int_{0}^{s} g(x, t, \lambda) d t$ and consider the $C^{1}$ functional $\tilde{\varphi}_{\lambda}: W^{1, p}(\Omega) \rightarrow \boldsymbol{R}$ defined by

$\tilde{\varphi}_{\lambda}(u)=\frac{1}{p}\|\nabla u\|_{p}^{p}+\frac{1}{p} \int_{\Omega} \beta|u|^{p} d x-\int_{\Omega} G(x, u, \lambda) d x \quad$ for all $u \in W^{1, p}(\Omega)$.

CLAIM 1. $\tilde{\varphi}_{\lambda}$ satisfies the Cerami condition.

Let $\left\{u_{k}\right\}_{k \geq 1} \subset W^{1, p}(\Omega)$ be a sequence such that

$$
\left|\tilde{\varphi}_{\lambda}\left(u_{k}\right)\right| \leq M_{3} \text { for all } k \geq 1,
$$

for some $M_{3}>0$, and

$$
\left(1+\left\|u_{k}\right\|\right) \tilde{\varphi}_{\lambda}^{\prime}\left(u_{k}\right) \rightarrow 0 \quad \text { in } \quad W^{1, p}(\Omega)^{*} \text { as } k \rightarrow \infty .
$$

We have to show that $\left\{u_{k}\right\}_{k \geq 1}$ admits a strongly convergent subsequence in $W^{1, p}(\Omega)$. From (28) we have

$$
\left.\left|\left\langle A\left(u_{k}\right), y\right\rangle+\int_{\Omega} \beta\right| u_{k}\right|^{p-2} u_{k} y d x-\int_{\Omega} g\left(x, u_{k}, \lambda\right) y d x \mid \leq \frac{\varepsilon_{k}\|y\|}{1+\left\|u_{k}\right\|}
$$

for all $y \in W^{1, p}(\Omega)$, with $\varepsilon_{k} \downarrow 0$. In (29) we choose $y=-u_{k}^{-} \in W^{1, p}(\Omega)$ and have

$$
\left\|\nabla u_{k}^{-}\right\|_{p}^{p}+\int_{\Omega} \beta\left(u_{k}^{-}\right)^{p} d x-\int_{\Omega} f\left(x, u_{0}, \lambda\right)\left(-u_{k}^{-}\right) d x \leq \varepsilon_{k} \quad \text { for all } k \geq 1 .
$$

This implies that $\hat{c}\left\|u_{k}^{-}\right\|^{p} \leq \varepsilon_{k}$ for all $k \geq 1$ (see (3)), which guarantees that

$$
\left\{u_{k}^{-}\right\}_{k \geq 1} \text { is bounded in } W^{1, p}(\Omega) \text {. }
$$

Next, in (29) we insert $y=u_{k}^{+} \in W^{1, p}(\Omega)$ and we deduce that

$$
-\left\|\nabla u_{k}^{+}\right\|_{p}^{p}-\int_{\Omega} \beta\left(u_{k}^{+}\right)^{p} d x+\int_{\Omega} g\left(x, u_{k}^{+}, \lambda\right) u_{k}^{+} d x \leq \varepsilon_{k} \quad \text { for all } k \geq 1 .
$$


On the other hand, (27) and (30) yield

$$
\left\|\nabla u_{k}^{+}\right\|_{p}^{p}+\int_{\Omega} \beta\left(u_{k}^{+}\right)^{p} d x-\int_{\Omega} p G\left(x, u_{k}^{+}, \lambda\right) d x \leq M_{4} \text { for all } k \geq 1,
$$

with $M_{4}>0$. Adding (31) and (32), and taking into account (26), we obtain

$$
\int_{\Omega}\left(f\left(x, u_{k}^{+}, \lambda\right) u_{k}^{+}-p F\left(x, u_{k}^{+}, \lambda\right)\right) d x \leq M_{5} \text { for all } k \geq 1,
$$

for some $M_{5}>0$. Reasoning as in the Claim in the proof of Proposition 3.3 by relying this time on (33) in place of (13), we show that $\left\{u_{k}^{+}\right\}_{k \geq 1}$ is bounded in $W^{1, p}(\Omega)$. This, together with (30), implies that $\left\{u_{k}\right\}_{k \geq 1}$ is bounded in $W^{1, p}(\Omega)$. Then, arguing as in the corresponding part of the proof of Proposition 3.3, we deduce that $\tilde{\varphi}_{\lambda}$ satisfies the Cerami condition.

CLAIM 2. We may assume that $u_{0}$ is a local $W^{1, p}(\Omega)$-minimizer of $\tilde{\varphi}_{\lambda}$.

We introduce the following truncation of $g(x, \cdot, \lambda)$ :

$$
\hat{g}(x, s, \lambda)= \begin{cases}g(x, s, \lambda) & \text { if } s \leq \hat{u}(x) \\ g(x, \hat{u}(x), \lambda) & \text { if } s>\hat{u}(x) .\end{cases}
$$

This is a Carathéodory function. Let $\hat{G}(x, s, \lambda)=\int_{0}^{s} \hat{g}(x, t, \lambda) d t$ and consider the $C^{1}$ functional $\hat{\psi}_{\lambda}: W^{1, p}(\Omega) \rightarrow \boldsymbol{R}$ defined by

$$
\hat{\psi}_{\lambda}(u)=\frac{1}{p}\|\nabla u\|_{p}^{p}+\frac{1}{p} \int_{\Omega} \beta|u|^{p} d x-\int_{\Omega} \hat{G}(x, u, \lambda) d x \text { for all } u \in W^{1, p}(\Omega) .
$$

It is clear that $\hat{\psi}_{\lambda}$ is coercive and sequentially weakly lower semicontinuous. So, we can find $\hat{u}_{0} \in W^{1, p}(\Omega)$ such that

$$
\hat{\psi}_{\lambda}\left(\hat{u}_{0}\right)=\inf \left\{\hat{\psi}_{\lambda}(u) ; u \in W^{1, p}(\Omega)\right\} .
$$

This yields $\hat{\psi}_{\lambda}^{\prime}\left(\hat{u}_{0}\right)=0$, that is,

$$
A\left(\hat{u}_{0}\right)+\beta\left|\hat{u}_{0}\right|^{p-2} \hat{u}_{0}=\hat{g}\left(\cdot, \hat{u}_{0}(\cdot), \lambda\right) .
$$

Acting on (35) with $\left(u_{0}-\hat{u}_{0}\right)^{+} \in W^{1, p}(\Omega)$, recalling that $u_{0} \leq \hat{u}$ and using (34), (26), and the fact that $u_{0}$ is solution of problem $\left(P_{\lambda}\right)$, we have

$$
\begin{aligned}
& \left\langle A\left(\hat{u}_{0}\right),\left(u_{0}-\hat{u}_{0}\right)^{+}\right\rangle+\int_{\Omega} \beta\left|\hat{u}_{0}\right|^{p-2} \hat{u}_{0}\left(u_{0}-\hat{u}_{0}\right)^{+} d x \\
& \quad=\int_{\Omega} \hat{g}\left(x, \hat{u}_{0}, \lambda\right)\left(u_{0}-\hat{u}_{0}\right)^{+} d x=\int_{\Omega} f\left(x, u_{0}, \lambda\right)\left(u_{0}-\hat{u}_{0}\right)^{+} d x \\
& \quad=\left\langle A\left(u_{0}\right),\left(u_{0}-\hat{u}_{0}\right)^{+}\right\rangle+\int_{\Omega} \beta u_{0}^{p-1}\left(u_{0}-\hat{u}_{0}\right)^{+} d x,
\end{aligned}
$$

which implies that $\left|\left\{u_{0}>\hat{u}_{0}\right\}\right|_{N}=0$, i.e., $u_{0} \leq \hat{u}_{0}$. Also, acting on (35) with $\left(\hat{u}_{0}-\hat{u}\right)^{+} \in$ $W^{1, p}(\Omega)$, and using (34), (26), the inequality $u_{0} \leq \hat{u}$, hypothesis $\left(\mathrm{H}_{f}\right)$ (vii), and the facts that $\lambda<\hat{\lambda}$ and $\hat{u}$ is solution of problem $\left(P_{\hat{\lambda}}\right)$, we obtain

$$
\left\langle A\left(\hat{u}_{0}\right),\left(\hat{u}_{0}-\hat{u}\right)^{+}\right\rangle+\int_{\Omega} \beta \hat{u}_{0}^{p-1}\left(\hat{u}_{0}-\hat{u}\right)^{+} d x=\int_{\Omega} \hat{g}\left(x, \hat{u}_{0}, \lambda\right)\left(\hat{u}_{0}-\hat{u}\right)^{+} d x
$$




$$
\begin{aligned}
& =\int_{\Omega} f(x, \hat{u}, \lambda)\left(\hat{u}_{0}-\hat{u}\right)^{+} d x \leq \int_{\Omega} f(x, \hat{u}, \hat{\lambda})\left(\hat{u}_{0}-\hat{u}\right)^{+} d x \\
& =\left\langle A(\hat{u}),\left(\hat{u}_{0}-\hat{u}\right)^{+}\right\rangle+\int_{\Omega} \beta \hat{u}^{p-1}\left(\hat{u}_{0}-\hat{u}\right)^{+} d x .
\end{aligned}
$$

It follows that $\left|\left\{\hat{u}_{0}>\hat{u}\right\}\right|_{N}=0$, i.e., $\hat{u}_{0} \leq \hat{u}$. Therefore, we have $\hat{u}_{0} \in\left[u_{0}, \hat{u}\right]$ and (35) becomes $A\left(\hat{u}_{0}\right)+\beta \hat{u}_{0}^{p-1}=f\left(\cdot, \hat{u}_{0}(\cdot), \lambda\right)$ (see (34) and (26)), so $\hat{u}_{0}$ solves problem $\left(P_{\lambda}\right)$. Using the nonlinear regularity theory we see that $\hat{u}_{0} \in C^{1}(\bar{\Omega})$. If $u_{0} \neq \hat{u}_{0}$, then $\hat{u}_{0}$ is a second positive solution of problem $\left(P_{\lambda}\right)$ and so we are done. Hence, we may assume that $u_{0}=\hat{u}_{0}$, that is, $u_{0}$ is a global minimizer of $\hat{\psi}_{\lambda}$. Recall that $u_{0}<\hat{u}$ in $\bar{\Omega}$ and note that $\left.\tilde{\varphi}_{\lambda}\right|_{[0, \hat{u}]}=$ $\left.\hat{\psi}_{\lambda}\right|_{[0, \hat{u}]}$. So, $u_{0}$ is a local $C^{1}(\bar{\Omega})$-minimizer of $\tilde{\varphi}_{\lambda}$, hence by virtue of [11, Proposition 24], $u_{0}$ is also a local $W^{1, p}(\Omega)$-minimizer of $\tilde{\varphi}_{\lambda}$. This proves Claim 2 .

Denote $B_{\rho}\left(u_{0}\right)=\left\{u \in W^{1, p}(\Omega) ;\left\|u-u_{0}\right\|<\rho\right\}$. Due to Claim 2, we can find $\rho_{0}>0$ such that $\tilde{\varphi}_{\lambda}\left(u_{0}\right)=\inf \left\{\tilde{\varphi}_{\lambda}(u) ; u \in B_{\rho_{0}}\left(u_{0}\right)\right\}$. Note that we may assume that

$$
\tilde{\varphi}_{\lambda}\left(u_{0}\right)<\tilde{\varphi}_{\lambda}(u) \text { for all } u \in B_{\rho_{0}}\left(u_{0}\right), u \neq u_{0} .
$$

Indeed, otherwise we find $\tilde{u}_{0} \in B_{\rho_{0}}\left(u_{0}\right), \tilde{u}_{0} \neq u_{0}$, with $\tilde{\varphi}_{\lambda}\left(\tilde{u}_{0}\right)=\tilde{\varphi}_{\lambda}\left(u_{0}\right)$. Then, $\tilde{u}_{0}$ is a minimizer of the restriction of $\tilde{\varphi}_{\lambda}$ to $B_{\rho_{0}}\left(u_{0}\right)$, hence a critical point of $\tilde{\varphi}_{\lambda}$. Writing explicitly the equality $\tilde{\varphi}_{\lambda}^{\prime}\left(\tilde{u}_{0}\right)=0$, a direct comparison by testing with $\left(u_{0}-\tilde{u}_{0}\right)^{+}$shows that $\tilde{u}_{0} \geq u_{0}$, $\tilde{u}_{0} \in C^{1}(\bar{\Omega})$ and so, by virtue of $(26), \tilde{u}_{0}$ is another positive solution of $\left(P_{\lambda}\right)$, and we are done. Fix $\rho \in\left(0, \rho_{0}\right)$.

Claim 3. We have $\tilde{\varphi}_{\lambda}\left(u_{0}\right)<\inf \left\{\tilde{\varphi}_{\lambda}(u) ;\left\|u-u_{0}\right\|=\rho\right\}=: \hat{\eta}_{\rho}$.

Arguing by contradiction, assume that there is a sequence $\left\{u_{k}\right\}_{k \geq 1} \subset W^{1, p}(\Omega), \| u_{k}-$ $u_{0} \|=\rho$, such that $\lim _{k \rightarrow \infty} \tilde{\varphi}_{\lambda}\left(u_{k}\right)=\tilde{\varphi}_{\lambda}\left(u_{0}\right)$. Up to passing to a subsequence, we may assume that $u_{k} \stackrel{\mathrm{w}}{\rightarrow} \tilde{u}_{1}$ in $W^{1, p}(\Omega)$, for some $\tilde{u}_{1} \in \overline{B_{\rho}\left(u_{0}\right)}$. Since $\tilde{\varphi}_{\lambda}$ is sequentially weakly lower semicontinuous, we get $\tilde{\varphi}_{\lambda}\left(\tilde{u}_{1}\right) \leq \tilde{\varphi}_{\lambda}\left(u_{0}\right)$, whence $\tilde{u}_{1}=u_{0}$ (see (36)). So, we have

$$
u_{k} \stackrel{\mathrm{w}}{\rightarrow} u_{0} \text { in } W^{1, p}(\Omega) \text { and } u_{k} \rightarrow u_{0} \text { in } L^{r}(\Omega) \text { as } k \rightarrow \infty \text { for all } r \in\left[1, p^{*}\right) .
$$

For each $k \geq 1$, the mean value theorem yields $t_{k} \in(0,1)$ such that

$$
\tilde{\varphi}_{\lambda}\left(u_{k}\right)-\tilde{\varphi}_{\lambda}\left(\frac{u_{k}+u_{0}}{2}\right)=\left\langle\tilde{\varphi}_{\lambda}^{\prime}\left(v_{k}\right), \frac{u_{k}-u_{0}}{2}\right\rangle
$$

where $v_{k}=t_{k} u_{k}+\left(1-t_{k}\right)\left(u_{k}+u_{0}\right) / 2$. Thus $v_{k} \stackrel{\text { w }}{\rightarrow} u_{0}$ in $W^{1, p}(\Omega)$ as $k \rightarrow \infty$. We have

$$
\begin{aligned}
& \left\langle A\left(v_{k}\right), v_{k}-u_{0}\right\rangle=\left(t_{k}+1\right)\left\langle A\left(v_{k}\right), \frac{u_{k}-u_{0}}{2}\right\rangle \\
& =\left(t_{k}+1\right)\left[\tilde{\varphi}_{\lambda}\left(u_{k}\right)-\tilde{\varphi}_{\lambda}\left(\frac{u_{k}+u_{0}}{2}\right)-\int_{\Omega}\left(\beta\left|v_{k}\right|^{p-2} v_{k}-g\left(x, v_{k}, \lambda\right)\right) \frac{u_{k}-u_{0}}{2} d x\right]
\end{aligned}
$$

for all $k \geq 1$ (see (38)). Using (37), the fact that $\tilde{\varphi}_{\lambda}$ is sequentially weakly lower semicontinuous, (26), and $\left(\mathrm{H}_{f}\right)(\mathrm{i})$, we deduce

$$
\limsup _{k \rightarrow \infty}\left\langle A\left(v_{k}\right), v_{k}-u_{0}\right\rangle \leq 0 .
$$


Since the operator $A$ is of type $(S)_{+}$, we obtain $v_{k} \rightarrow u_{0}$ in $W^{1, p}(\Omega)$ as $k \rightarrow \infty$. However

$$
\left\|v_{k}-u_{0}\right\|=\frac{1+t_{k}}{2}\left\|u_{k}-u_{0}\right\| \geq \frac{\rho}{2} \text { for all } k \geq 1,
$$

which is contradictory. This proves Claim 3.

Hypothesis $\left(\mathrm{H}_{f}\right)(\mathrm{ii})$ and (26) imply

$$
\tilde{\varphi}_{\lambda}(\theta) \rightarrow-\infty \text { as } \theta \rightarrow+\infty, \theta \in \boldsymbol{R} .
$$

Then Claim 3, (39) and Claim 1 permit the use of the mountain pass theorem (see, e.g., [5, Corollary 5.2.7]). We obtain $\tilde{u} \in W^{1, p}(\Omega)$ such that

$$
\tilde{\varphi}_{\lambda}\left(u_{0}\right)<\hat{\eta}_{\rho} \leq \tilde{\varphi}_{\lambda}(\tilde{u})
$$

(see Claim 3) and

$$
\tilde{\varphi}_{\lambda}^{\prime}(\tilde{u})=0 .
$$

Relation (40) entails $\tilde{u} \neq u_{0}$. From (41) and (26) we have $u_{0} \leq \tilde{u}$ and $A(\tilde{u})+\beta \tilde{u}^{p-1}=$ $f(\cdot, \tilde{u}(\cdot), \lambda)$. Using the nonlinear regularity theory, we note that $\tilde{u} \in C^{1}(\bar{\Omega})$. So $\tilde{u}$ is a second positive solution of $\left(P_{\lambda}\right)$.

\section{Examples of nonlinearities satisfying $\left(\mathrm{H}_{f}\right)$.}

(a) A typical example of function satisfying hypotheses $\left(\mathrm{H}_{f}\right)$ (for the sake of simplicity we drop the $x$-dependence) is

$$
f(s, \lambda)=\lambda s^{q-1}+s^{r-1} \text { for all } s \geq 0, \text { all } \lambda>0 \text {, with } 1<q<p<r<p^{*} .
$$

So, our assumptions incorporate problems with a combination of concave and convex terms.

(b) However, our hypotheses allow to go beyond the classical situation of concaveconvex nonlinearities: a more general example of function satisfying hypotheses $\left(\mathrm{H}_{f}\right)$ is

$$
f(s, \lambda)=\lambda s^{q(\lambda)-1}+s^{r(\lambda)-1} \text { for all } s \geq 0, \text { all } \lambda>0,
$$

where $q:(0,+\infty) \rightarrow(1, p)$ and $r:(0,+\infty) \rightarrow\left(\tilde{p}, p^{*}\right)$ (with some $\left.\tilde{p}>p\right)$ are derivable functions such that $r^{\prime}(\lambda)=-\lambda q^{\prime}(\lambda) \geq 0$ for all $\lambda \in(0,+\infty)$.

(c) Another function satisfying hypotheses $\left(\mathrm{H}_{f}\right)$ is

$$
f(s, \lambda)=\lambda \min \left\{1, s^{q(\lambda)-1}\right\}+s^{r-1} \quad \text { for all } s \geq 0, \text { all } \lambda>0,
$$

where $q:(0,+\infty) \rightarrow(1, p)$ is a nonincreasing, derivable function and $r \in\left(p, p^{*}\right)$.

(d) More generally, the following function satisfies $\left(\mathrm{H}_{f}\right)$ :

$$
f(s, \lambda)=\left\{\begin{array}{ll}
\lambda s^{q(s, \lambda)-1}+s^{r-1} & \text { if } s \in[0,1), \\
\lambda+s^{r-1} & \text { if } s \in[1,+\infty),
\end{array} \quad \text { for all } \lambda>0,\right.
$$

with $r \in\left(p, p^{*}\right)$ and $q:[0,1) \times(0,+\infty) \rightarrow[1, p)$ continuous such that

- for each $s \in[0,1), \lambda \mapsto q(s, \lambda)$ is nonincreasing, derivable on $(0,+\infty)$;

- for each $\lambda \in(0,+\infty), q(0, \lambda)>1$ and $s \mapsto q(s, \lambda)$ is derivable on $(0,1)$ with bounded from above derivative. 
(e) Other examples of nonlinearities which are not of concave-convex type are those with two branches whose dividing point depends on $\lambda$. For instance, the following function

$$
f(s, \lambda)=\left\{\begin{array}{ll}
\lambda s^{q-1}+s^{r-1} & \text { if } s \in[0, \lambda), \\
\lambda^{q}+s^{r-1} & \text { if } s \in[\lambda,+\infty),
\end{array} \quad \text { for all } \lambda>0,\right.
$$

where $1<q<p<r<p^{*}$, satisfies hypotheses $\left(\mathrm{H}_{f}\right)$.

(f) Finally, an example of function satisfying hypotheses $\left(\mathrm{H}_{f}\right)$ which does not fulfill the Ambrosetti-Rabinowitz condition is

$$
f(s, \lambda)=\left\{\begin{array}{ll}
\lambda\left(s^{q(\lambda)-1}-c s^{\theta(\lambda)-1}\right) & \text { if } \quad s \in[0,1), \\
s^{p-1} \ln s+\lambda(1-c) & \text { if } \quad s \in[1,+\infty),
\end{array} \quad \text { for all } \lambda>0,\right.
$$

where $c \in[0,1)$ and $q, \theta:(0,+\infty) \rightarrow(1,+\infty)$ are derivable functions satisfying $q(\lambda)<$ $\min \{p, \theta(\lambda)\}$ and $q^{\prime}(\lambda) \leq \min \left\{0, c \theta^{\prime}(\lambda)\right\}$ for all $\lambda \in(0,+\infty)$.

\section{REFERENCES}

[ 1 ] S. Aizicovici, N. S. Papageorgiou And V. Staicu, The spectrum and an index formula for the Neumann $p$-Laplacian and multiple solutions for problems with a crossing nonlinearity, Discrete Contin. Dyn. Syst. 25 (2009), 431-456.

[2] A. Ambrosetti, H. Brezis and G. Cerami, Combined effects of concave and convex nonlinearities in some elliptic problems, J. Funct. Anal. 122 (1994), 519-543.

[ 3 ] A. Ambrosetti And P. H. Rabinowitz, Dual variational methods in critical point theory and applications, J. Funct. Anal. 14 (1973), 349-381.

[ 4 ] J. P. García Azorero, I. Peral Alonso and J. J. Manfredi, Sobolev versus Hölder local minimizers and global multiplicity for some quasilinear elliptic equations, Commun. Contemp. Math. 2 (2000), 385404.

[ 5 ] L. Gasiński and N. S. Papageorgiou, Nonlinear analysis, Ser. Math. Anal. Appl. 9, Chapman \& Hall/CRC, Boca Raton, 2006.

[6] Z. GUO AND Z. ZHANG, $W^{1, p}$ versus $C^{1}$ local minimizers and multiplicity results for quasilinear elliptic equations, J. Math. Anal. Appl. 286 (2003), 32-50.

[ 7 ] S. HU AND N. S. PAPAGEORGIOU, Multiplicity of solutions for parametric $p$-Laplacian equations with nonlinearity concave near the origin, Tohoku Math. J. 62 (2010), 137-162.

[ 8 ] N. Kenmochi, Pseudomonotone operators and nonlinear elliptic boundary value problems, J. Math. Soc. Japan 27 (1975), 121-149.

[ 9 ] G. M. Lieberman, Boundary regularity for solutions of degenerate elliptic equations, Nonlinear Anal. 12 (1988), 1203-1219.

[10] G. LI AND C. YANG, The existence of a nontrivial solution to a nonlinear elliptic boundary value problem of $p$-Laplacian type without the Ambrosetti-Rabinowitz condition, Nonlinear Anal. 72 (2010), 4602-4613.

[11] S. Miyajima, D. Motreanu and M. Tanaka, Multiple existence results of solutions for the Neumann problems via super- and sub-solutions, J. Funct. Anal. 262 (2012), 1921-1953.

[12] D. Motreanu And N. S. PApageorgiou, Existence and multiplicity of solutions for Neumann problems, J. Differential Equations 232 (2007), 1-35.

[13] N. S. PAPAgeorgiou AND G. SMYRlis, Positive solutions for nonlinear Neumann problems with concave and convex terms, Positivity 16 (2012), 271-296. 
DÉPARTEMENT de MATHÉMATIQUes UNIVERSITÉ DE PERPIGNAN 66860 PERPIGNAN

FRANCE

E-mail address: motreanu@univ-perp.fr

Department of Mathematics

NATIONAL TECHNICAL UNIVERSITY ATHENS 15780

GREECE

E-mail address: npapg@math.ntua.gr
DEPARTMENT OF MATHEMATICS

BEN GURION UNIVERSITY OF THE NEGEV BE'ER SHEVA 84105

ISRAEL

E-mail address: motreanu@ post.bgu.ac.il 